\title{
Effect of liposomal phosphatidylcholine acyl chain length on the bactericidal activity of liposome encapsulated nisin on cariogenic streptococcus
}

\section{mutans}

\begin{abstract}
Background: Dental caries is an infectious oral disease caused by cariogenic Streptococcus mutans. The streptococci inhabit dental-biofilms which comprise insoluble glucans.

Objectives: To prevent dental caries, liposome-encapsulated nisin prepared from phosphatidylcholines with different acyl chain lengths were tested for bactericidal activity against cariogenic $S$. mutans.

Materials and Methods: Liposomal nisins prepared from dimyristoylphosphatidylcholine (DMPC), dipalmitoylphosphatidylcholine (DPPC), and distearoyl phoshatidylcholine (DSPC) were tested for their bactericidal activity against S. mutans 10449 and as a consequence, to inhibit the synthesis of insoluble glucan by the streptococci in vitro.

Results: The bactericidal activities by minimum inhibitory concentration assay, DSPCnisin, DPPC-nisin, and DMPC-nisin were higher by factors of 10, 3.3, and 2.5 compared with unencapsulated nisin. Inhibition of glucan synthesis was sustained the longest by DSPC-nisin. On the release assay of these liposomes, nisin was released at the highest and lowest rates from DMPC-nisin and DSPC-nisin, respectively. The highest and most sustained levels of bactericidal activity correlated with the slow release of encapsulated nisin
\end{abstract}

Conclusion: The DSPC-nisin may be useful for preventing the formation of dental caries due to sustained release of nisin. Thus, prophylaxis with the liposomal nisin may serve to maintain oral health.

Keywords: Nisin, liposome, phosphatidylcholine, acyl chain, streptococcus mutans, dental caries
Volume I Issue 3 - 2014

\author{
Kazuo Yamakami,' Hideaki Tsumori, ${ }^{2}$ \\ Yoshitaka Shimizu, ${ }^{3}$ Yutaka Sakurai,' Kohei \\ Nagatoshi, ${ }^{4}$ Kenji Sonomoto ${ }^{5}$ \\ 'Department of Preventive Medicine and Public Health, National \\ Defense Medical College, Japan \\ 2Department of Chemistry, National Defense Medical College, \\ Japan \\ ${ }^{3}$ Research \& Development Division, BioMedCore Inc., Japan \\ ${ }^{4}$ Eco Friendly Institute Ltd., Japan \\ ${ }^{5}$ Department of Bioscience and Biotechnology, Faculty of \\ Agriculture, Graduate School, Kyushu University, Japan
}

Correspondence: Kazuo Yamakami, Department of Preventive Medicine and Public Health, National Defense Medical College, Tokorozawa 359-85 I J Japan, Tel 8I-4-2995-1563, Email yamakami@ndmc.ac.jp

Received: June 10,2014 | Published: June 18, 2014
Abbreviations: DMPC, Dimyristoylphosphatidylcholine;
DPPC, Dipalmitoyl-phosphatidylcholine; Distearoylphosphatidylcholine; FDEL, freeze-dried empty liposomes; MIC, minimum inhibitory concentration; THB, todd hewitt broth

\section{Introduction}

Infectious oral diseases associated with the synthesis of insoluble glucan-biofilm, such as dental caries, afflict the majority of the world's population. Insoluble glucans on tooth surfaces mediate bacterial colonization, and are an essential element in the pathogenesis of dental caries. ${ }^{1}$ Cariogenic streptococci contribute to the synthesis of insoluble glucan in the oral cavity, and these polysaccharides contribute to the formation of tartar, plaque, and dental caries. ${ }^{2}$ Agents that exert sustained bactericidal activity against Streptococcus mutans may contribute to maintaining dental health. Therefore, focuses on the development of agents that kill $S$. mutans and inhibit consequently glucan formation, because any inhibitor of glucan-biofilm synthesis should effectively prevent dental caries. Nisin is a class I bacteriocin produced by Lactococcus lactis subsp. lactis. ${ }^{3}$ It has been found to inhibit the viability of Gram-positive bacteria. Bactericidal activity of nisin against $S$. mutans leads to inhibition of insoluble glucan formation, and nisin does not appear to be toxic to normal human gingival fibroblasts and epithelial cells. ${ }^{4,5}$ Furthermore, Tong et al. ${ }^{6}$ investigated the role of nisin in oral health. However, unencapsulated nisin is degraded by enzymatic and nonenzymatic reactions. ${ }^{3}$ The advantages of liposomes include maintaining the sustained activity of molecules that they encapsulate and releasing them on demand. ${ }^{7,8}$ However, the outcome of our efforts to effectively encapsulate nisin in liposomes was not encouraging. For the purpose of prevention of dental caries, we investigated the effect of acyl chain lengths of liposomal nisin against cariogenic streptococci.

\section{Material and methods}

\section{Preparation of liposome-encapsulated nisin}

Nisin was prepared from Lactococcus lactis subsp. lactis ATCC 11454 (American Type Culture Collection; Manassas, VA, USA) and was purified as described previously. ${ }^{3,9}$ Liposomes were prepared using the freeze-dried empty liposomes (FDEL) method. ${ }^{10}$ Phospholipids with the same headgroup but different lengths of saturated carbon chains were as follows: dimyristoylphosphatidylcholine (DMPC), dipalmitoylphosphatidylcholine (DPPC) and distearoylphosphatidylcholine (DSPC) (Avanti Polar Lipids. Inc., Alabaster, AL, USA). To prepare liposomal nisin, $1 \mathrm{ml}$ of nisin $(3.0$ $\mathrm{nmol}$ ) in $5 \mathrm{mM}$ sodium citrate ( $\mathrm{pH} 5.0$ ) was added to the FDEL (40 nmol), which were vortexed at $30^{\circ} \mathrm{C}, 46^{\circ} \mathrm{C}$, and $60^{\circ} \mathrm{C}$ for DMPC, 
DPPC, and DSPC, respectively. To determine the efficiency of encapsulation, $20 \mu 1$ of liposomes were mixed with $80 \mu 1$ of an aqueos solution of $2 \%$ Triton X-100 and incubated at $37^{\circ} \mathrm{C}$ to release nisin. Release efficiency was determined by comparing the amounts of starting and released nisin using CBQCA protein assay kit (Molecule Probes, Eugene, OR, USA).

\section{Assay of bactericidal activity of liposome-encapsulated nisin}

Determinations of the minimum inhibitory concentrations (MIC) of liposomal or unencapsulated nisin preparations against $S$. mutans were performed using the broth dilution method. ${ }^{3}$ The nisin preparations were added to the wells of microplates at final concentrations of 4.0 to $40 \mathrm{pmol}$ in $50 \mu \mathrm{l}$ of $10 \mathrm{mM}$ sodium citrate (pH 7.0). S. mutans ATCC 10449 in 50 $\mu 1$ of Todd Hewitt Broth (THB; Invitrogen, Carlsbad, CA, USA) were added to the wells, the plates were incubated at $37^{\circ} \mathrm{C}$ overnight and then assayed for absorbance at $600 \mathrm{~nm}$. The MIC values were defined as the lowest concentrations of unencapsulated and encapsulated nisin that inhibited the growth of $S$. mutans. Combinations of unencapsulated nisin plus individual empty liposomes served as the control.

\section{Assay of inhibitory effect of liposome-encapsulated nisin on insoluble glucan synthesis}

To determine the effects of liposomal nisin on the synthesis of insoluble glucan-biofilm, insoluble glucan was generated using $S$. mutans cultured in microplates. Assays were performed in a solution containing S. mutans in $50 \mu$ l of THB, $50 \mu 1$ of $1.0 \%$ sucrose, and bacteriocin (unencapsulated nisin, $30 \mathrm{pmol}$; and liposomal nisin, 30 pmol as the nisin equivalent), or a mixture of unencapsulated nisin plus an appropriate empty liposome in $100 \mu 1$ of $5 \mathrm{mM}$ sodium citrate $(\mathrm{pH} 7.0)$ were placed in each well. The plates were incubated at $37^{\circ} \mathrm{C}$, and insoluble glucans were quantified at appropriate times. Insoluble glucans in each well were washed with $10 \mathrm{mM}$ sodium phosphate buffer ( $\mathrm{pH} 7.0$ ) and suspended in $200 \mu \mathrm{l}$ of $1.0 \mathrm{M}$ sodium hydroxide. Solubilized glucans were quantified by the phenol-sulfuric acid method. ${ }^{11}$ Inhibition of glucan synthesis was determined according to the amounts of glucan-biofilm in each well compared with those in wells lacking nisin or liposomes.

\section{In vitro nisin release assay from liposome-encapsulated nisin}

In vitro assays of nisin release from the liposomes were performed by incubating liposomal nisin (100 pmol nisin equivalent) in $200 \mu 1$ of $5 \mathrm{mM}$ Tris- $\mathrm{HCl}(\mathrm{pH} 7.0)$ containing $0.02 \%$ Tween 20 at $37^{\circ} \mathrm{C}$. At the appropriate times, the supernatants obtained by centrifuging the liposomes at 20,000xg for $10 \mathrm{~min}$ were assayed fluorometrically using the CBQCA quantitation kit. The amounts of nisin are expressed as the cumulative percentage of nisin released at each time.

\section{Statistical analysis}

Assays were performed in triplicate, and values are reported as the mean. The standard deviation (SD) was $<5 \%$ for all assays. The statistical significance of differences between groups was assessed using the Student t-test, and $\mathrm{P}<0.05$ was considered statistically significant.

\section{Results and discussion}

Because we are trying to develop liposomal nisin as prophylaxis for dental caries, we examined the bactericidal activity of the encapsulated nisin against cariogenic streptococci. DMPC, DPPC, and DSPC were used to generate of liposomes loaded with nisin. The encapsulation efficiency of nisin in liposomes was investigated. DPPC liposomes were most efficiently loaded with nisin accounted for the highest efficiency (80\%), followed by DSPC and DMPC liposomes $(76 \%$ each). The difference may be due to the variability of the preparations. On encapsulation efficiencies of nisin of these liposomes, composing phospholipids with the same polar headgroups but with different length of saturated hydrocarbon chains had nearly the same values. ${ }^{12}$ In this regard Anderson and Omri ${ }^{13}$ indicated that the encapsulation efficiency is influenced by the interactions between positively-charged molecules and the same zwitterionic headgroup of phospholipids. Evaluation of liposomal nisin was assessed by bactericidal activity against the streptococci. The MIC value of unencapsulated nisin was $20 \mathrm{nmol}$ after $16 \mathrm{~h}$ of incubation with $S$. mutans as the target microorganism compared with 8,6 , and $2 \mathrm{nmol}$ for DMPC, DPPC, and DSPC-nisin, respectively (Figure 1). Thus, the bactericidal activity of DSPC-nisin was highest at the lowest dose. Empty liposomes did not inhibit bacterial growth.

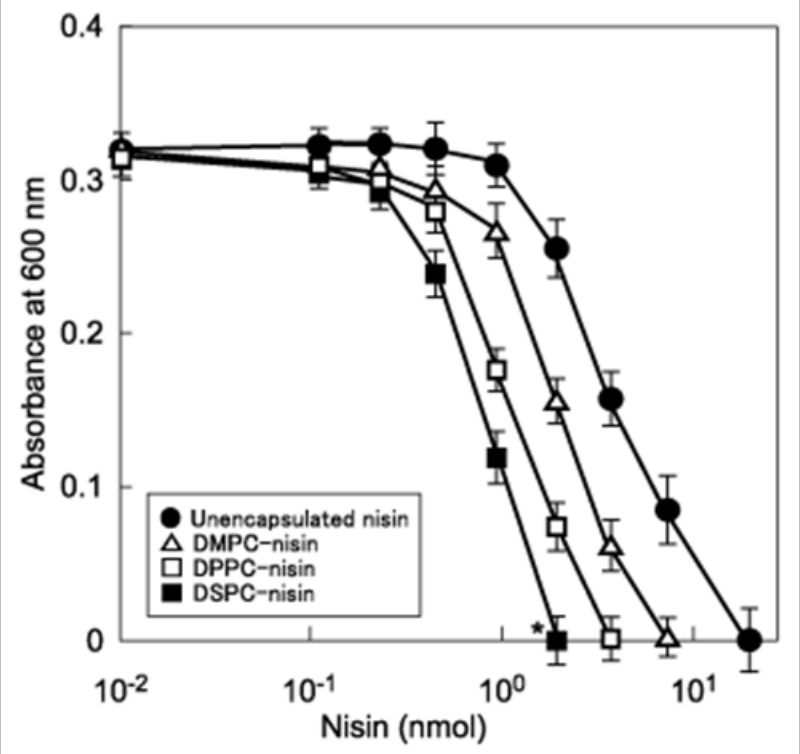

Figure I Bactericidal activity of liposome-encapsulated nisin against Streptococcus mutans. Exponentially proliferating cultures of S. mutans 10449 (absorbance of 0.02 at $600 \mathrm{~nm}$ ) were incubated overnight at $37^{\circ} \mathrm{C}$ in THB in the presence of liposomal nisin, a nisin-equivalent concentration of unencapsulated nisin, or buffer. $* \mathrm{P}<0.05$ indicates a significant difference of MIC values of the treated cultures compared with the control of buffer alone. Values represent the mean of triplicate assays and the bars represent the SD.

On the assessment of efficiency of bactericidal activity of above liposomes, we assayed the inhibition of glucan-biofilm synthesis by $S$. mutans in vitro. The inhibitory effects of liposomal nisin on insoluble glucan synthesis by $S$. mutans are shown in Figure 2. Unencapsulated and encapsulated nisin preparations caused a significant delay in glucan synthesis compared with controls $(\mathrm{P}<0.05)$, and DPPC and DSPCnisin delayed glucan synthesis greater than unencapsulated nisin for up to $4.5 \mathrm{~h}$, and the increase was exponential. There was no significant difference between the inhibitory effects of DMPC-nisin and DPPCnisin. DSPC-nisin delayed glucan synthesis the longest, which was greater by a factor of 2.5 compared with unencapsulated nisin at $4.5 \mathrm{~h}$ $(\mathrm{P}<0.05)$. However, empty liposomes had no significant effect. These findings indicate that the acyl chain length of phosphatidylcholine mediated the sustained bactericidal activity of encapsulated nisin. Liposomal nisin composed of DSPC exerted the highest bactericidal 
activity as compared to those containing DPPC and DMPC. The finding may indicates that the liposome composed of DSPC can sustain the bioactivity of nisin. Bactericidal activity paralleled the inhibition of insoluble glucan formation, which is critically important for preventing periodontal disease. It is desirable that the inhibition of glucan synthesis of liposomal nisin be sustain so that a selected formulation would suffice to achieve an efficient preventive effect for oral health. ${ }^{4,11}$ Thus, the stable bactericidal activity and long lasting inhibition of glucan synthesis of liposomes will allow us to have expected the dental health.

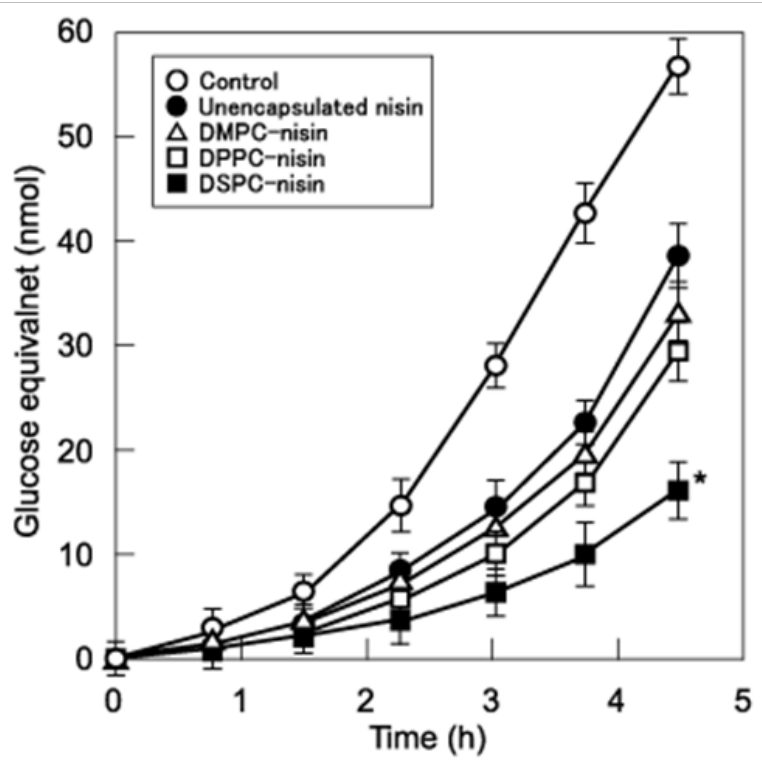

Figure 2 Effect of liposomal nisin on insoluble glucan-biofilm synthesis by Streptococcus mutans. Exponentially proliferating cultures of S. mutans 10449 (absorbance of 0.02 at $600 \mathrm{~nm}$ ) were incubated in THB containing $0.25 \%$ sucrose at $37^{\circ} \mathrm{C}$ for $6 \mathrm{~h}$ in the presence of liposomal nisin at a concentration equivalent to that of unencapsulated nisin, a combination of corresponding amounts of empty liposomes plus unencapsulated nisin, or unencapsulated nisin. *P $<0.05$ compared with the group of buffer alone. Values represent the mean of triplicate assays and bars represent the SD.

We assessed the data of bactericidal activity and inhibition of glucan synthesis of these encapsulated nisin, therefore we investigated the nisin releasing assay of these liposomes (Figure 3). In our assay conditions, nisin was slowly released by DSPC-nisin for up to $60 \mathrm{~min}$, and its level gradually increased to $50 \%$ for the next $60 \mathrm{~min}$. DPPCnisin released nisin at an intermediate during the 120 min incubation. The DMPC-nisin showed a quick release of nisin, through the first 30 min, on that time point, an increase in its release after 90 min with the values of $80 \%$ was observed. These values were significantly different at $120 \mathrm{~min}$. The lipid formulations of liposomes may influence the release of encapsulated nisin. ${ }^{14}$ Furthermore, nisin releasing profiles are proportional to the acyl chain length of phosphphatidylcholine. DMPC, DPPC and DSPC have longer saturated acyl chains $(14,16$, and 18 carbons, respectively) and their phase transition temperatures increase according to the chain length, which is consistent with our finding here that DSPC-nisin retained the highest retention of nisin in our liposomes. ${ }^{13}$ The relative rate of release of nisin from vesicles were DMPC $>$ DPPC $>$ DSPC, which reflects the fluidity of their respective vesicles, acyl chain length, and phase-transition temperatures, respectively. ${ }^{14}$ With regard to the efficiency of liposomal nisin, the selection of acyl chain length of phospholipids is quite evident from our finding. It has been postulated that liposome stabilization is increased with long acyl groups which explain why DSPC-nisin releases nisin slowly more than that of DPPC-nisin or DMPC-nisin. ${ }^{12}$ The present study shows that by varying the lipid formulation, nisinrelease profiles from the liposomes could be generated depending on the length of acyl chain of phospholipids. The findings indicate that the hydrophobic interactions between positively charged nisin and the acyl chain of phospholipids induce sustained bactericidal activity.

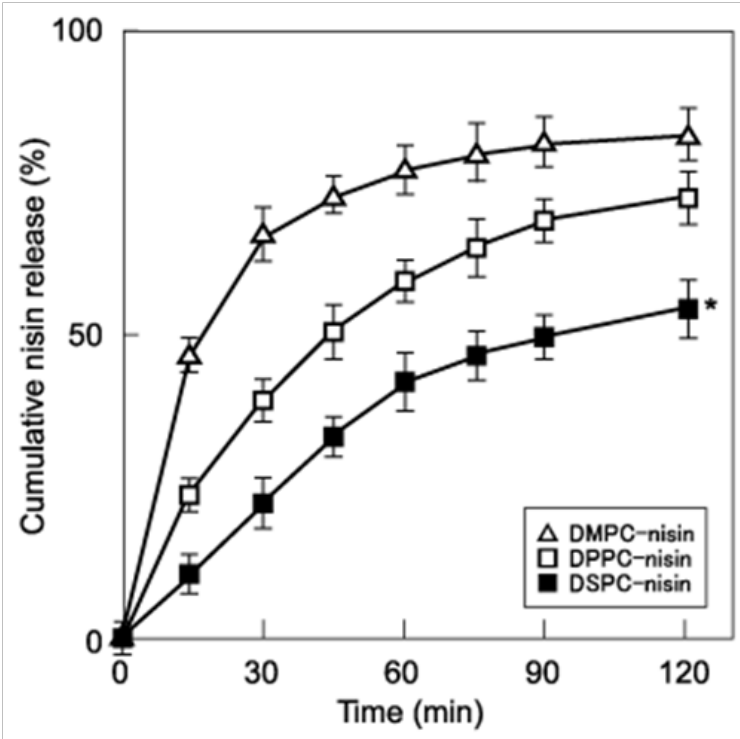

Figure 3 Analysis of the release of nisin from liposomes in vitro. Liposomal nisin was incubated in Tris- $\mathrm{HCl}\left(\mathrm{pH} \mathrm{7.0)}\right.$ containing $0.02 \%$ Tween 20 at $37^{\circ} \mathrm{C}$ and the supernatants were assayed over time.The levels of nisin are expressed as the cumulative percentage released.The maximal value was defined as $100 \%$ release. The assays were performed in triplicate at each time, and the bars represent the SD. $* \mathrm{P}<0.05$ compared with the group of DMPC-nisin.

\section{Conclusion}

The DSPC-nisin may be useful for preventing the formation of dental caries due to sustained release of nisin. Our consideration in development of liposome formulations is the long lasting bactericidal activity against cariogenic streptococci. Therefore, our future studies are focused on the development of nisin-encapsulated liposomes in order to ascertain the preventive potential for maintaining oral health.

\section{Acknowledgments}

None.

\section{Conflict of interest}

The authors declare that there is no conflict of interest.

\section{References}

1. Cury JA, Rebelo MA, Del Bel Cury AA, et al. Biochemical composition and cariogenicity of dental plaque formed in the presence of sucrose or glucose and fructose. Caries Res. 2000;34(6):491-497.

2. Paes Leme AF, Koo H, Bellato CM, et al. The role of sucrose in cariogenic dental biofilm formation--new insight. J Dent Res. 2006;85(10):878-887.

3. Kuwano K, Tanaka N, Shimizu T, et al. Dual antibacterial mechanisms of nisin $\mathrm{Z}$ against Gram-positive and Gram-negative bacteria. Int $J$ Antimicrob Agents. 2005;26(5):396-402. 
4. Yamakami K, Tsumori H, Sakurai Y, et al. Sustainable inhibition efficacy of liposome-encapsulated nisin on insoluble glucan-bilfilm synthesis by Streptococcus mutans. Pharm Biol. 2013;51(2):267-270.

5. Akerey B, Le-Lay C, Fliss I, et al. In vitro efficacy of nisin Z agains Candida albicans adhesion and transition following contact with normal human gingival cells. J Appl Microbiol. 2009;107(4):1298-1307.

6. Tong Z, Dong L, Zhou L, et al. Nisin inhibits dental caries-associated microorganism in vitro. Peptides. 2010;31(11):2003-2008.

7. Ikegami S, Yamakami K, Ono T, et al. Targeting gene therapy for prostate cancer cells by liposomes complexed with anti-prostate-specific membrane antigen monoclonal antibody. Human Gene Ther. 2006;17(10):997-1005.

8. Matsui M, Shimizu Y, Kodera Y, Kondo E, et al. Targeted delivery of oligomannose-coated liposome to the omental micrometastasis by peritoneal macrophages from patients with gastric cancer. Cancer Sci. 2010;101(7):1670-1677.
9. Field D, Connor PM, Cotter PD, et al. The generation of nisin variants with enhanced activity against specific gram-positive pathogens. Mol Microbiol. 2008;69(1):218-230.

10. Kikuchi H, Suzuki N, Ebihara K, et al. Gene delivery using liposome technology. J Control Release. 1999;62(1-2):269-277.

11. Tsumori H, Shimamura A, Sakurai Y, et al. Inhibitory effects of mutanase and dextranase on the formation of insoluble glucan-biofilms by cariogenic streptococci. Food Func. 2012;10(1):37-42.

12. Daghastanli KR, Ferreira RB, Thedei G Jr, et al. Lipid compositiondependent incorporation of multiple membrane proteins into liposomes. Colloids Surf B Biointerfaces. 2004;36(3-4):127-137.

13. Anderson M, Omri A. The effect of different lipid components on the in vitro stability and release kinetics of liposome formulations. Drug Delivery. 2004;11(1):33-39.

14. Barenholz Y. Liposome application: problems and prospects. Curr Opin Colloid Interface Sci. 2001;6(1):66-77. 